05

\title{
Высокоэффективная схема перераспределения оптического излучения на пространственных решетках атомных населенностей
}

\author{
() М.Ю. Гордеев, Ю.В. Рождественский \\ Университет ИТМО, \\ 197101 Санкт-Петербург, Россия \\ e-mail: mxmgordeev@gmail.com
}

Поступила в редакцию 04.12.2018 г.

В окончательной редакции 18.03.2019 г.

Принята к публикации 22.03.2019 г.

\begin{abstract}
Теоретически исследовано перераспределение интенсивности поля пробного излучения при рассеянии на пространственно периодических решетках атомных населенностей в среде с четырехуровневой трипод-конфигурацией атомных состояний. Найдены условия, при которых возникает существенное перераспределение интенсивности поля пробной волны, и формируется „дифракционная““ картина с эффективной перекачкой интенсивности пробного поля в максимумы первого порядка.
\end{abstract}

Ключевые слова: электромагнитно индуцированная решетка, трипод-конфигурация

DOI: $10.21883 /$ OS.2019.07.47942.358-18

\section{Введение}

Пионерские теоретические [1] и экспериментальные [2] исследования эффекта электромагнитно индуцированных решеток (ЭМИР) вызывают существенный интерес, обусловленный потенциальными возможностями использования такого эффекта в оптических устройствах быстрого переключения света [3,4], хранения света [5,6] в настраиваемых фотонных ячейках [7], а также других приложений [8-13]. При этом для получения эффекта могут использоваться различные методы, такие как микроволновая модуляция одного из переходов атомной системы [14], использование гигантской керровской нелинейности [15-17], усиление спонтанно генерируемой когерентности [18], получение максимальной атомной когерентности [19], контроль спонтанного излучения атомов [20].

Подчеркнем, что оптические устройства на основе ЭМИР делают возможным переключение света на скоростях порядка мегагерц, что не может быть обеспечено существующими электромеханическими приборами. Причем помимо высокой скорости такие устройства обладают еще и компактностью, что играет существенную роль при конструировании оптических систем. Таким образом, именно сочетание высокой скорости переключения направления распространения света и компактности делают привлекательными для исследования устройства на основе эффекта ЭМИР.

Наиболее интересной с практической точки зрения является трипод-схема, которая представляет из себя классическую $\Lambda$-схему с добавлением одного дополнительного нижнего уровня и соответствующего разрешенного перехода с верхнего уровня. Дополнительный разрешенный переход позволяет вводить в систему больший набор параметров, создающих возможность для большего контроля системы и вариативности ис- пользования параметров. Эти параметры могут влиять, например, на тип создаваемой ЭМИР - амплитудной, фазовой или амплитудно-фазовой. Однако наличие большого набора параметров требует и более глубокого анализа системы и ее ключевых характеристик. Так, анализ выражений для коэффициентов преломления и поглощения позволяет правильно выбрать параметры системы для создания высокоэффективного и в то же время компактного устройства на основе ЭМИР в среде с трипод-конфигурацией атомов.

В работе [21] была исследована возможная схема создания ЭМИР в среде с трипод-конфигурацией атомных состояний. Однако предложенная схема и продемонстрированные результаты исследования не могут быть применены для создания оптических устройств. Попробуем оценить предполагаемый размер ячейки со средой, используя параметры из работы [21]. Физический размер ячейки зависит от двух параметров:

$$
z_{0}=\frac{2 \hbar \gamma_{41} \varepsilon_{0}}{N k_{p} \mu_{41}^{2}} \quad \text { и } \quad L=z / z_{0}
$$

где $z_{0}-$ резонансная длина поглощения поля в $e$ раз под действием модулирующих полей, $\hbar-$ постоянная Планка, $\gamma_{41}$ - скорость спонтанного распада населенности, $\varepsilon_{0}$ - диэлектрическая проницаемость вакуума, $N$ - атомная плотность, $k_{p}$ - волновое число пробного поля, $\mu_{41}$ - дипольной момент, $L-$ толщина ячейки со средой в единицах $z_{0}, z-$ физический размер ячейки вдоль оси $Z$. Вычислим физическую длину ячейки

$$
\begin{gathered}
z=L \frac{2 \hbar \gamma_{41} \varepsilon_{0}}{N k_{p} \mu_{41}^{2}} \approx 1.5 m, \\
L=900, \\
\hbar=1.055 \cdot 10^{-34} \mathrm{~J} \cdot \mathrm{s},
\end{gathered}
$$




$$
\begin{gathered}
\gamma_{41} \approx 10^{-6} \mathrm{~s}^{-1}, \\
\varepsilon_{0}=8.854 \cdot 10^{-12} \mathrm{~m}^{-3} \cdot \mathrm{kg}^{-1} \cdot \mathrm{s}^{4} \cdot \mathrm{A}^{2}, \\
N \approx 10^{19}, \\
k_{p}=\frac{1}{\lambda_{p}} \approx 0.2 \cdot 10^{7} \mathrm{~m}^{-1}, \\
\mu_{41} \approx 2.5 \cdot 10^{-37} \mathrm{C} \cdot \mathrm{m}^{-2} .
\end{gathered}
$$

Как видно из результата вычислений, трудно говорить о компактности устройства, основанного на использовании ЭМИР, в исследованной в работе [21] схеме.

С другой стороны, большие физические размеры исследованной в работе [21] схемы говорят также о малой эффективности такого устройства. Понимание причин лежит в анализе подобранных параметров. Например, резонансно настроенные модулирующие поля и нерезонансное взаимодействие пробного поля со средой говорят о том, что не используется эффект электромагнитно индуцированной прозрачности (ЭИП), в результате чего пробное поле сильно поглощается средой. Как следствие, при компактных размерах ячейки не наблюдается эффективного перераспределения интенсивности пробного поля в максимумы первого порядка.

В настоящей работе рассмотрена возможность создания ЭМИР в среде с трипод-конфигурацией атомных уровней. Выбор схемы не случаен, а обусловлен тем, что, в отличие от трехуровневой $\Lambda$-схемы, четырехуровневая трипод-схема позволяет создавать двумерные и даже трехмерные решетки атомных населенностей, что может существенно увеличить эффективную перекачку энергии в максимумы первого порядка. Кроме того, существенным преимуществом данной схемы является сравнительная простота реализации экспериментов со средой в трипод-конфигурации атомных уровней. В качестве такой среды может быть использован, к примеру, метастабильный гелий Не или рубидий ${ }^{85} \mathrm{Rb}$.

Структура работы следующая: в разд. 1 представлена теоретическая схема и базовые уравнения, на основании которых исследуется возможность создания ЭМИР в четырехуровневой трипод-схеме. В разд. 2 представлены результаты исследований на основании теории, изложенной в разд. 1. Выводы представлены в разд. 3.

\section{1. Основные уравнения}

Принципиальная схема моделируемого эксперимента продемонстрирована на рис. $1, a$. Две сильные стоячие волны $\Omega_{1}$ и $\Omega_{3}$ действуют вдоль оси $O x$ и модулируют среду. Пробная бегущая волна $\Omega_{2}$ распространяется перпендикулярно к ним по оси $O z$. На выходе из ячейки со средой наблюдается перераспределение в интенсивности прошедшего пучка.

Энергетическая схема атомных уровней в триподконфигурации представлена на рис. $1, b$. На переходе $|2\rangle-|4\rangle$ атомной системы вдоль направления $O z$ действует поле пробной бегущей волны с частотой Раби $\Omega_{2}$ и отстройкой $\Delta_{2}$ от резонансного значения. Поля, действующие на переходах $|1\rangle-|4\rangle$ и $|3\rangle-|4\rangle$, направлены вдоль оси $O x$ и имеют круговую поляризацию в противоположных направлениях. На переходе $|1\rangle-|4\rangle$ действует поле сильной стоячей волны с пространственнозависимой частотой Раби $\Omega_{1}(x)$ и отстройкой $\Delta_{1}$ от резонансного значения, а на переходе $|3\rangle-|4\rangle-$ сильное поле стоячей волны $\Omega_{3}(x)$ с отстройкой $\Delta_{3}$ от резонанса. Оптические релаксации по каналам $|4\rangle-|n\rangle(n=1,2,3)$ определяются соответственно константами $\gamma_{1}, \gamma_{2}$ и $\gamma_{3}$. Тогда естественная ширина уровня $|4\rangle$ будет равна $2 \gamma=\gamma_{1}+\gamma_{2}+\gamma_{3}$. Полуширины оптических переходов обозначены как $\Gamma_{14}, \Gamma_{24}$ и $\Gamma_{34}$. Помимо этого, возможен распад когерентностей между нижними состояниями атома (релаксации низкочастотных когерентностей) со скоростями, равными $\Gamma_{12}, \Gamma_{13}$ и $\Gamma_{23}$.

В результате поле, модулирующее атомы в среде, запишем в виде

$$
\mathbf{E}_{1,3}=\mathbf{e}_{1} E_{1} \cos \left(\omega_{1} t-k_{1} x\right)+\mathbf{e}_{3} E_{3} \cos \left(\omega_{3} t+k_{3} x\right),
$$

в то время как поле пробной волны имеет вид

$$
\mathbf{E}_{2}=\mathbf{e}_{2} E_{2} \cos \left(\omega_{2} t-k_{2} z\right),
$$

где волна с $\omega_{1}, k_{1}$ распространяется в положительном и волна с $\omega_{3}, k_{3}$ в отрицательном направлении оси $O x$, а пробная волна с $\omega_{2}, k_{2}$ распространяется в положительном направлении оси $O z$. При этом векторы $\mathbf{e}_{1,3}$ задают круговую поляризацию в положительном и отрицательном направлении соответственно, а вектор $\mathbf{e}_{2}$ определяет линейную поляризацию.

Отметим, что в упомянутой выше работе [21] конфигурация полей в системе отличается от описанной в настоящей работе. В частности, пробное поле в нашем случае действует на переходе $|2\rangle-|4\rangle$, в то время как в работе [21] пробное поле действует на переходе $|1\rangle-|4\rangle$ четырехуровневой трипод-системы.

Для исследования возможностей управления пространственным перераспределением пробного поля световой волны на ЭМИР используем систему самосогласованных уравнений, которая состоит из уравнений Лиувилля для элементов матрицы плотности в приближении вращающейся волны и дипольном приближении, которые описывают состояние среды, и укороченного волнового уравнения в приближении медленно меняющихся амплитуд, описывающего распространение пробного поля в среде.

Уравнение Лиувилля, описывающее динамику состояний атомов, имеет вид

$$
i \hbar \dot{\tilde{\rho}}=[H, \tilde{\rho}]_{i j}+i \Gamma_{i j} \tilde{\rho}_{i j},
$$

с гамильтонианом в представлении взаимодействия в виде $H=H_{0}+V$, где $H_{0}$ задает внутреннее состояние системы без возмущения, а $V=-\frac{1}{\hbar} \sum_{n=1}^{3}\left(\mathbf{d}_{n 4} \cdot \mathbf{e}_{n}\right) E_{n}$ определяет взаимодействие с полем оптического излучения $(1 \mathrm{a}, \mathrm{b})$ для переходов $|n\rangle-|4\rangle,(n=1,2,3)$ с 

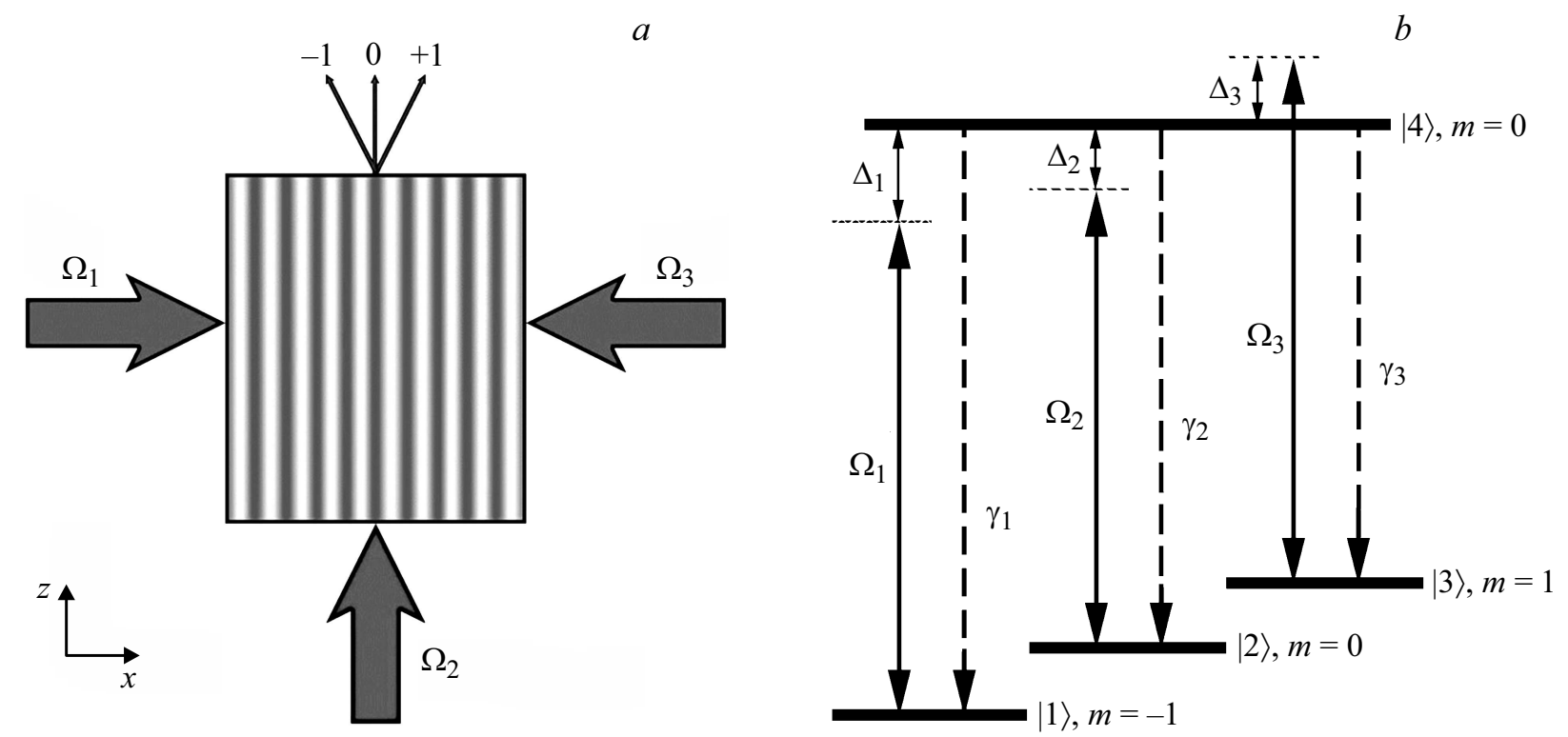

Рис. 1. (a) Принципиальная схема эксперимента; (b) Энергетическая схема атомных уровней в трипод-конфигурации.

матричным элементом оператор дипольного взаимодействия $\mathbf{d}_{k 4}$.

В выражении (2) матрица $\Gamma_{i j}$ задает скорости релаксации элементов $\tilde{\rho}_{i j}(x, t)$. При этом скорость релаксации диагональных матричных элементов (т.е. населенностей) определяется естественной шириной $2 \gamma$ верхнего возбужденного состояния системы (рис. $1, b$ ) $2 \gamma=\gamma_{1}+\gamma_{2}+\gamma_{3}$, а скорости релаксации недиагональных матричных элементов $\Gamma_{i j}(i \neq j)$ могут наряду со скоростью естественного распада учитывать и другие возможные механизмы уширения (столкновения, конечную ширину спектра возбуждающих полей и пр.).

С учетом обозначенных выше приближений уравнение Лиувилля (2) сводится к системе уравнений для элементов матрицы плотности $\rho_{i j}(x, y, t)$ четырехуровневого атома следующего вида:

$$
\begin{gathered}
i \dot{\rho}_{11}=\Omega_{1}\left(\rho_{14}-\rho_{41}\right)+i \gamma_{1} \rho_{44}, \\
i \dot{\rho}_{22}=\Omega_{2}\left(\rho_{24}-\rho_{42}\right)+i \gamma_{2} \rho_{44}, \\
i \dot{\rho}_{33}=\Omega_{3}\left(\rho_{34}-\rho_{43}\right)+i \gamma_{3} \rho_{44}, \\
i \dot{\rho}_{44}=\Omega_{1}\left(\rho_{41}-\rho_{14}\right)+\Omega_{2}\left(\rho_{42}-\rho_{24}\right) \\
+\Omega_{3}\left(\rho_{43}-\rho_{34}\right)-i\left(\gamma_{1}+\gamma_{2}+\gamma_{3}\right) \rho_{44}, \\
i \dot{\rho}_{14}=\Omega_{1}\left(\rho_{11}-\rho_{44}\right)+\Omega_{2} \rho_{12}+\Omega_{3} \rho_{13}+\left(\Delta_{1}-i \Gamma_{14}\right) \rho_{14}, \\
i \dot{\rho}_{24}=\Omega_{2}\left(\rho_{22}-\rho_{44}\right)+\Omega_{1} \rho_{21}+\Omega_{3} \rho_{23}+\left(\Delta_{2}-i \Gamma_{24}\right) \rho_{24}, \\
i \dot{\rho}_{34}=\Omega_{3}\left(\rho_{33}-\rho_{44}\right)+\Omega_{1} \rho_{31}+\Omega_{2} \rho_{32}+\left(\Delta_{3}-i \Gamma_{34}\right) \rho_{34}, \\
i \dot{\rho}_{12}=\Omega_{2} \rho_{14}-\Omega_{1} \rho_{42}+\left(\Delta_{1}-\Delta_{2}\right) \rho_{12}, \\
i \dot{\rho}_{13}=\Omega_{3} \rho_{14}-\Omega_{1} \rho_{43}+\left(\Delta_{1}-\Delta_{3}\right) \rho_{13}, \\
i \dot{\rho}_{23}=\Omega_{3} \rho_{24}-\Omega_{2} \rho_{43}+\left(\Delta_{2}-\Delta_{3}\right) \rho_{23},
\end{gathered}
$$

где $\rho_{i j}=\rho_{j i}^{*}$, частоты Раби $\Omega_{n}=\frac{\left(\mathbf{d}_{n 4} \cdot \mathbf{e}_{n}\right) E_{n}}{2 \hbar}$. Считаем, что система замкнута $\rho_{11}+\rho_{22}+\rho_{33}+\rho_{44}=1$, а также бу- дем считать, что распадов низкочастотных когерентностей в системе нет по сравнению с другими распадами системы $\Gamma_{12}=\Gamma_{13}=\Gamma_{23}=0$.

При выводе системы уравнений (3) пренебрежем членами, содержащими временные осцилляции на удвоенной оптической частоте (резонансное приближение), и используем так называемое приближение вращающейся волны, которое состоит в замене недиагональных матричных элементов для оптических когерентностей $\tilde{\rho}_{n 4}=\rho_{n 4} e^{i \Delta_{n} t}(n=1,2,3)$ и для низкочастотных когерентностей $\tilde{\rho}_{12}=\rho_{12} e^{i\left(\Delta_{1}-\Delta_{2}\right) t}, \quad \tilde{\rho}_{13}=\rho_{13} e^{i\left(\Delta_{1}-\Delta_{3}\right) t}$, $\tilde{\rho}_{23}=\rho_{23} e^{i\left(\Delta_{2}-\Delta_{3}\right) t}$.

Воспользуемся волновым уравнением Максвелла для описания распространенияпробного поля в среде:

$$
\Delta E_{2}+\frac{1}{c^{2}} \frac{\partial^{2}}{\partial t^{2}} E_{2}=\frac{4 \pi}{c^{2}} \frac{\partial^{2}}{\partial t^{2}} P_{2}
$$

С использованием приближения медленно меняющихся амплитуд, в стационарном режиме уравнение сводится к следующему виду:

$$
-\frac{i}{2 k_{2}} \frac{\partial^{2} E_{02}}{\partial x^{2}}+\frac{\partial E_{02}}{\partial z}=i \frac{4 \pi k_{2}}{2 \varepsilon_{0}} P_{02}
$$

где $E_{02}$ - медленно меняющаяся амплитуда пробного поля, $c$ - скорость света в вакууме, $\varepsilon_{0}$ - диэлектрическая проницаемость вакуума. Используя усреднение дипольного момента на ансамбле однородно уширенной среды, получаем выражение для поляризации среды в виде

$$
P_{02}=2 N d_{24} \rho_{24},
$$

где $N$ - атомарная плотность, $d_{24}-$ проекция дипольного момента перехода $|2\rangle-|4\rangle$ на единичный вектор поляризации среды, $\rho_{24}$ - элемент матрицы плотности, когерентность перехода $|2\rangle-|4\rangle$. 
Для получения аналитических выражений вещественной и мнимой частей элемента $\rho_{24}$ используем стационарное решение уравнений (3), которое может быть получено в пренебрежении временными производными в левых частях по сравнению с членами в правых частях, содержащих скорости распада. Отметим, что в полученных выражениях были учтены члены всех порядков для модулирующих полей $\Omega_{1}(x), \Omega_{3}(x)$, но только линейные члены для поля пробной волны $\Omega_{2}$.

Подставим это решение в уравнение (6) и заменим поляризацию в уравнении (5) на получившееся после подстановки выражение. Для того чтобы получить окончательное выражение в безразмерной форме, выразим все распады, отстройки и частоты через $\gamma_{1}$, а координаты выразим через $\Lambda_{x}$ для оси $O x$ и $z_{0}$ для оси $O z$, где $\Lambda_{x}=\pi / k_{x}-$ пространственный период наведенной электромагнитно индуцированной решетки, $k_{x}$ - волновой вектор сильных стоячих волн, а $z_{0}$ имеет вид

$$
z_{0}=\frac{2 \hbar \gamma_{1} \varepsilon_{0}}{N k_{2} d_{24}^{2}}
$$

Тогда уравнение для поля приобретает вид

$$
-i \frac{\partial^{2} \Omega_{2}}{N_{F} \partial x^{2}}+\frac{\partial \Omega_{2}}{\partial z}=\left(\alpha_{r}+i \alpha_{i}\right) \Omega_{2},
$$

где $\alpha_{r}$ и $\alpha_{i}-$ коэффициенты преломления и поглощения пробного поля

$$
\begin{gathered}
\alpha_{r}=\frac{\Delta_{12} \Delta_{23}\left(\Omega_{3}^{2}(x) \Delta_{12}-\Omega_{1}^{2}(x) \Delta_{23}-\Delta_{2} \Delta_{12} \Delta_{23}\right)}{3 \Delta_{12}^{2} \Delta_{23}^{2} \gamma^{2}+\left(\Omega_{3}^{2}(x) \Delta_{12}-\Omega_{1}^{2}(x) \Delta_{23}-\Delta_{2} \Delta_{12} \Delta_{23}\right)}, \\
\alpha_{i}=\frac{3 \gamma \Delta_{12}^{2} \Delta_{23}^{2}}{6 \Delta_{12}^{2} \Delta_{23}^{2} \gamma^{2}+2\left(\Omega_{3}^{2}(x) \Delta_{12}-\Omega_{1}^{2}(x) \Delta_{23}-\Delta_{2} \Delta_{12} \Delta_{23}\right)^{2}}, \\
\Delta_{i j}=\Delta_{i}-\Delta_{j},
\end{gathered}
$$

а $N_{F}$ - число щелей ширины $2 \sqrt{\pi} \Lambda_{x}$, умещающихся на расстоянии $z_{0}$,

$$
N_{F}=\frac{\left(2 \sqrt{\pi} \Lambda_{x}\right)^{2}}{\lambda_{2} z_{0}} \text {. }
$$

Отметим, что полученные в явном виде аналитические выражения (9) для показателей преломления и поглощения пробного поля позволяют привести в следующем разделе данной работы качественный анализ параметров системы, влияющих на эффективное перераспределение интенсивности пробного поля, что, однако, не было сделано в работе [21].

Решая получившееся уравнение на поле, находим значение на выходе. Отношение поля на выходе $\Omega_{2 \text { out }}$

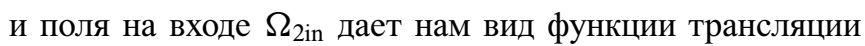
среды толщиной $L$ (по оси $O z$ ) по отношению к полю:

$$
T(x)=e^{\alpha_{r}(x) L} e^{i \alpha_{i}(x) L},
$$

где $T(x)=\Omega_{\text {2out }} / \Omega_{2 \text { in }}, L-$ толщина среды вдоль оси $O z$ в безразмерных единицах длины $z_{0}$.
Рассматривая вклад только дальнего поля (дифракция Фраунгофера) и считая, что волна пробного поля плоская и имеет одинаковую амплитуду поперек пучка ширины $M \Lambda_{x}$, можем определить интенсивность $I_{\text {out }}(\theta)$ как

$$
I_{\text {out }}(\theta)=\left|\Omega_{2}^{1}(\theta)\right|^{2} \frac{\sin ^{2}\left(M \pi \Lambda_{x} \sin \theta / \lambda_{2}\right)}{M^{2} \sin ^{2}\left(\pi \lambda_{x} \sin \theta / \lambda_{2}\right)},
$$

где

$$
\Omega_{2}^{1}(\theta)=\int_{0}^{1} T(x) \exp \left[-i 2 \pi \Lambda_{x} x \sin \theta / \lambda_{2}\right] d x
$$

Используя получившиеся аналитические выражения, исследуем зависимость интенсивности пробного поля на выходе $I_{\text {out }}$ из среды от синуса угла $\theta$.

\section{2. Обсуждение результатов}

Используя теорию, изложенную в предыдущем разделе, обратимся к исследованию возможности создания и эффективности ЭМИР в среде с трипод-конфигурацией атомных уровней в зависимости от различных параметpoв.

Вводя в систему резонансно $\Delta_{1}=\Delta_{3}=0$ модулирующие поля $\Omega_{1}$ и $\Omega_{3}$, наблюдаем распределение интенсивности пробного поля на выходе в виде одного максимума нулевого порядка без перераспределения интенсивности в максимумы высших порядков (рис. 2,a). При увеличении интенсивности модулирующих полей распределение не изменяется, увеличивается лишь нормированная интенсивность максимума нулевого порядка, которая в пределе стремится к 1 . Объясняется это тем, что условие $\Delta_{1}=\Delta_{3}=0$, как видно из (9), задает зависимости коэффициентов поглощения $\alpha_{i}$ и преломления $\alpha_{r}$ пробного поля константами. В результате на выходе из среды виден классический профиль гауссова пучка пробного поля.

В работе [21], однако, при резонансно действующих модулирующих полях с $\Delta_{1}=\Delta_{3}=0$ получено существенное перераспределение интенсивности пробного поля в максимумы первого и второго порядков. Фактически это означает, что такое перераспределение формируется не за счет классических физических механизмов, используемых при создании ЭМИР, а путем простого подбора одного из параметров системы, в частности отвечающего за физический размер ячейки $L$. Какими механизмами получено такое перераспределение на длине $1.5 \mathrm{~m}$ также сложно утверждать, как и предполагать стабильность и контролируемость такой системы.

Аналитические исследования выражений (9) дают условия для получения максимальной эффективности перераспределения интенсивности пробного поля $\Omega_{2}$ в виде

$$
\text { 1) } \frac{\Delta_{1}}{\Delta_{3}} \approx \frac{\Delta_{3}}{\Delta_{1}}
$$

2) $\Delta_{1}, \Delta_{3} \ll \Omega_{1}, \omega_{3}$. 


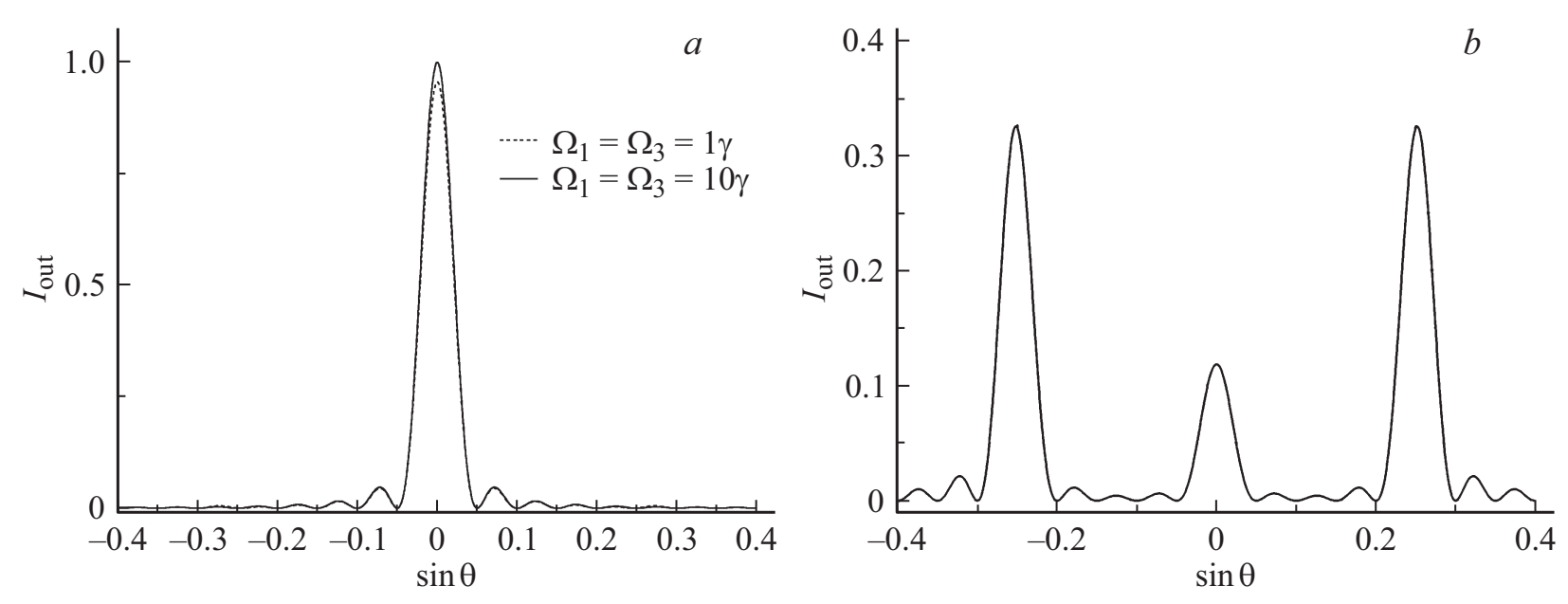

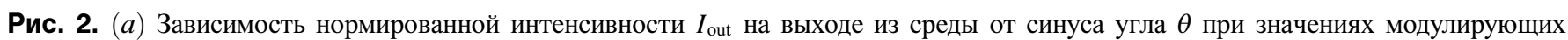
полей $\Omega_{1}=\Omega_{3}=\gamma$ (штриховая линия) и $10 \gamma$ (сплошная линия). Остальные параметры системы $\gamma_{1}=\gamma_{2}=\gamma_{3}=\gamma, \Delta_{1}=0, \Delta_{2}=0$, $\Delta_{3}=0, \varphi=0, L=7, \Lambda_{x} / \lambda_{2}=0.25, N=5$; $(b)$ Зависимость нормированной интенсивности $I_{\text {оut }}$ на выходе из среды от синуса угла $\theta$ при $\gamma_{1}=\gamma_{2}=\gamma_{3}=\gamma, \Omega_{1}=\Omega_{2}=\gamma, \Delta_{1}=0.05 \gamma, \Delta_{2}=0, \Delta_{3}=0.15 \gamma, \varphi=0, L=7, \Lambda_{x} / \lambda_{2}=0.75, N=5$.
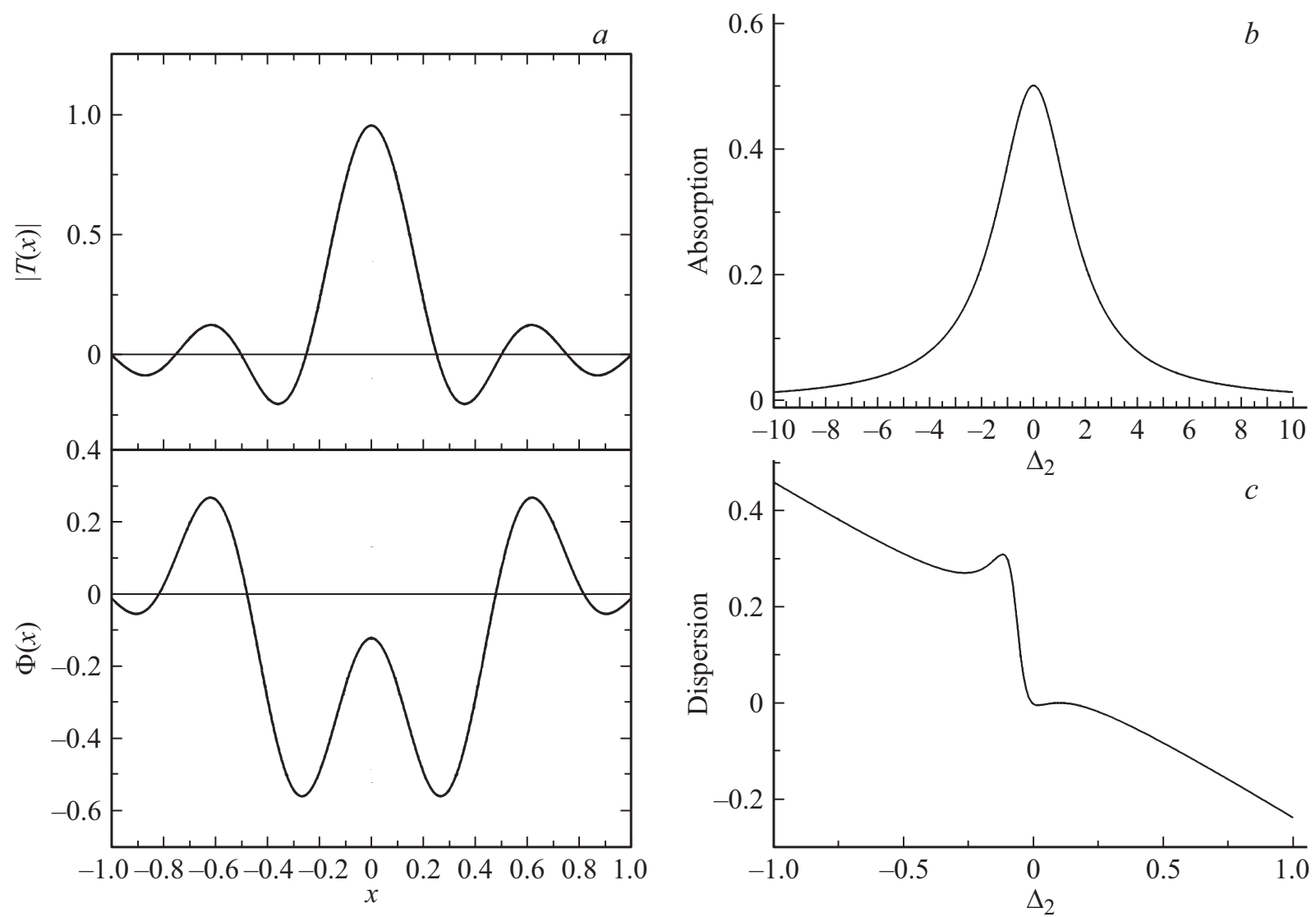

Pис. 3. (a) Зависимость интеграла Фраунгофера по амплитуде $|T(x)|$ и фазе $\Phi(x)$ трансляционной функции от пространственной координаты $X$ на одном пространственном периоде решетки. Остальные параметры системы $\gamma_{1}=\gamma_{2}=\gamma_{3}=\gamma, \Omega_{1}=\Omega_{2}=\gamma$, $\Delta_{1}=0.05 \gamma, \Delta_{2}=0, \Delta_{3}=0.15 \gamma, \varphi=0, L=7, \Lambda_{x} / \lambda_{2}=0.25, N=5$. Спектры поглощения (b) и преломления (c) пробного поля. Остальные параметры системы $\gamma_{1}=\gamma_{2}=\gamma_{3}=\gamma, \Omega_{1}=\Omega_{2}=\gamma, \Delta_{1}=0.05 \gamma, \Delta_{3}=0.15 \gamma, \varphi=0, L=7, \Lambda_{x} / \lambda_{2}=0.25, N=5$.

Физически полученные ограничения соответствуют условиям наличия в системе эффекта ЭИП, который в свою очередь создает окно прозрачности для пробного поля $\Omega_{2}$, позволяя проходить максимально возможной интенсивности без потерь. С другой стороны, данные условия формируют специфическую пространственную 

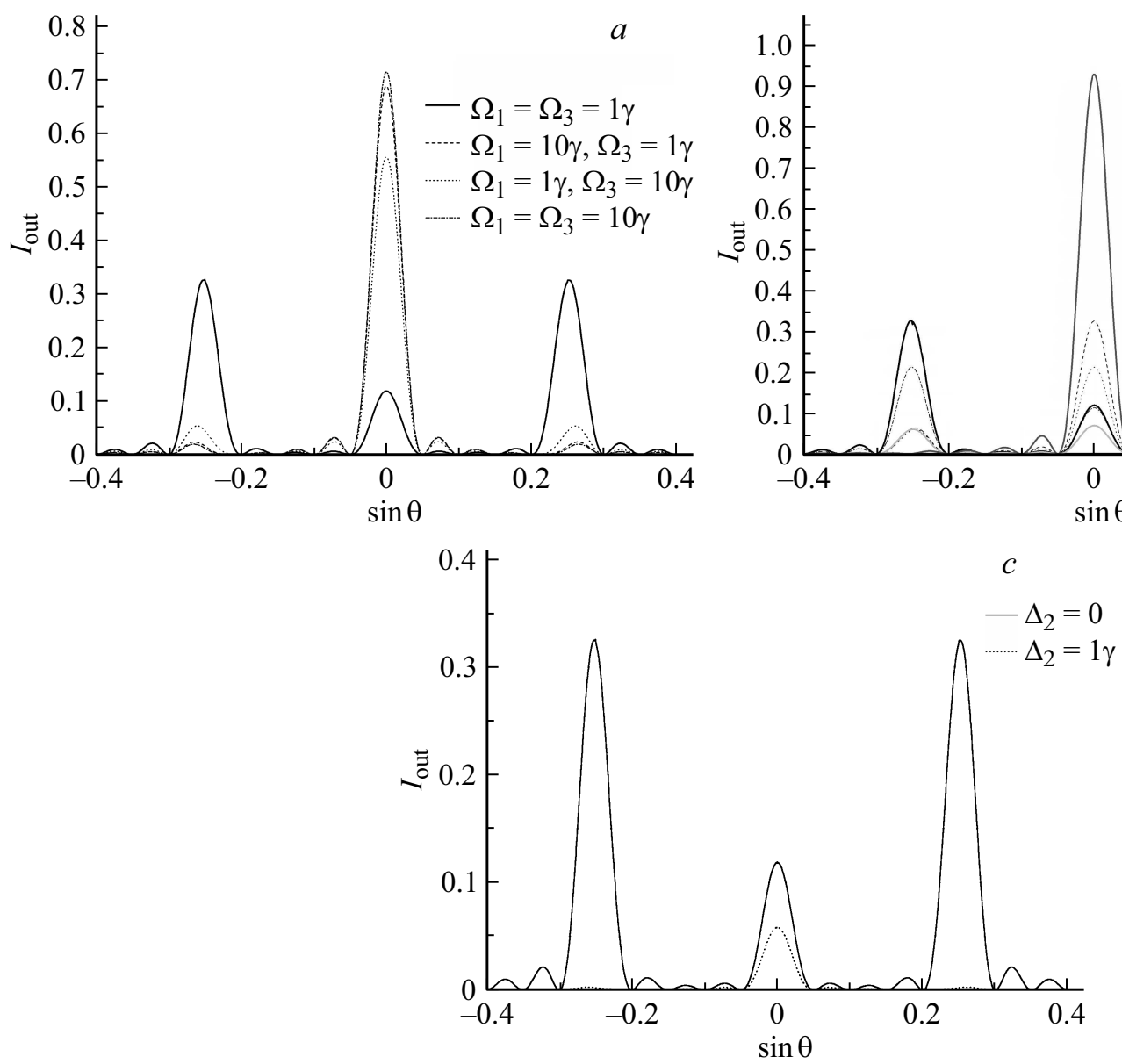

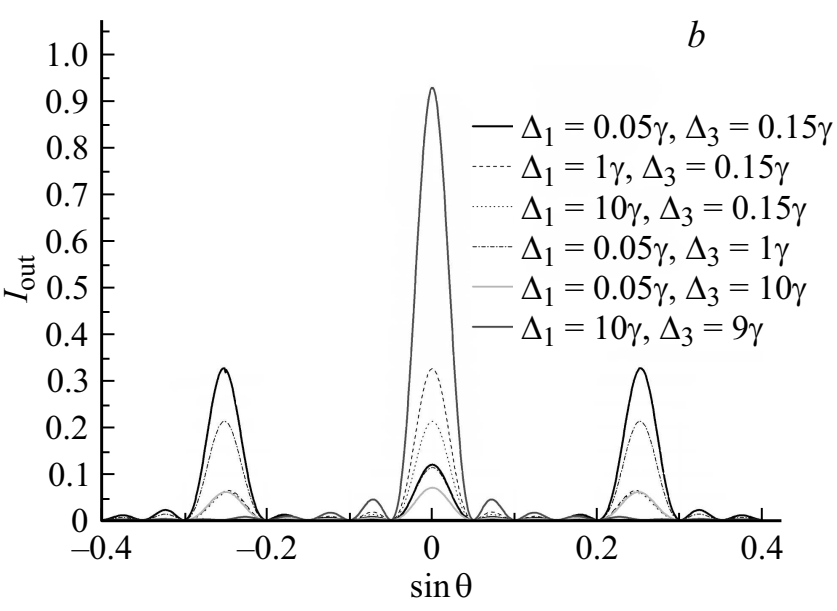

$\sin \theta$

Рис. 4. (a) Зависимость нормированной интенсивности $I_{\text {out }}$ на выходе из среды от синуса угла $\theta$ при различных значениях модулирующих полей $\Omega_{1}$ и $\Omega_{3}$. Остальные параметры системы $\gamma_{1}=\gamma_{2}=\gamma_{3}=\gamma, \Delta_{1}=0.05 \gamma, \Delta_{2}=0, \Delta_{3}=0.15 \gamma, \varphi=0, L=7$, $\Lambda_{x} / \lambda_{2}=0.25, N=5$. (b) Зависимость нормированной интенсивности $I_{\text {out }}$ на выходе из среды от синуса угла $\theta$ при различных значениях отстроек модулирующих полей $\Delta_{1}$ и $\Delta_{3}$. Остальные параметры системы $\gamma_{1}=\gamma_{2}=\gamma_{3}=\gamma, \Omega_{1}=\Omega_{3}=\gamma, \Delta_{2}=0, \varphi=0$, $L=7, \Lambda_{x} / \lambda_{2}=0.25, N=5$. (c) Зависимость нормированной интенсивности $I_{\text {out }}$ на выходе из среды от синуса угла $\theta$ при значениях отстройки пробного поля $\Delta_{2}=0$ (сплошная линия) и $\gamma$ (пунктир). Остальные параметры системы $\gamma_{1}=\gamma_{2}=\gamma_{3}=\gamma, \Omega_{1}=\Omega_{3}=\gamma$, $\Delta_{1}=0.05 \gamma, \Delta_{3}=0.15 \gamma, \varphi=0, L=7, \Lambda_{x} / \lambda_{2}=0.25, N=5$.

модуляцию профилей коэффициентов поглощения $\alpha_{i}$ и преломления $\alpha_{r}$ пробного поля $\Omega_{2}$, позволяющую достигнуть существенной эффективности перераспределения интенсивности падающего поля в максимумы высших порядков.

В результате становится возможным получение порядка 70\% интенсивности пробного поля $\Omega_{2}$ в максимумах первого порядка (рис. 2,b). Физический размер ячейки при этом не превышает $1 \mathrm{~cm}$, что говорит о высокой эффективности такой системы и компактных размерах.

Как было указано выше, условия (14) существенно влияют как на возможность получения перераспределенной интенсивности пробного поля, так и на эффективность этого распределения в максимумы высших порядков. Известно, что существует несколько типов ЭМИР, среди которых наиболее исследованными являются амплитудные и фазовые ЭМИР. Для понимания того, с каким типом ЭМИР имеем дело в данной работе, были получены дифракционные картины для амплитуды $|T(x)|$ и фазы $\exp [i \Phi(x)]$ функции трансляции (рис. 3, $a$ ).

На рис. 3, $a$ представленызависимости интеграла Фраунгофера по амплитуде функции трансляции $|T(x)|$ и по фазе функции трансляции $\exp [i \Phi(x)]$. Видно, что амплитудная часть перераспределяет интенсивность преимущественно в центральный максимум, в то время как фазовая часть перенаправляет свет в первые порядки. Таким образом, в нашей системе возможно говорить об амплитудно-фазовом типе решетки с преобладанием фазовой модуляции.

Обратимся теперь к исследованиям параметров системы на примере полученных зависимостей перераспределения интенсивности пробного поля $\Omega_{2}$ на выходе из среды. Как уже было отмечено при рассмотрении случая $\Delta_{1}=\Delta_{3}=0$, увеличение значений модулирующих полей $\Omega_{1}$ и $\Omega_{3}$ не приводит к перераспределению интенсивности пробного поля в максимумы высших по- 


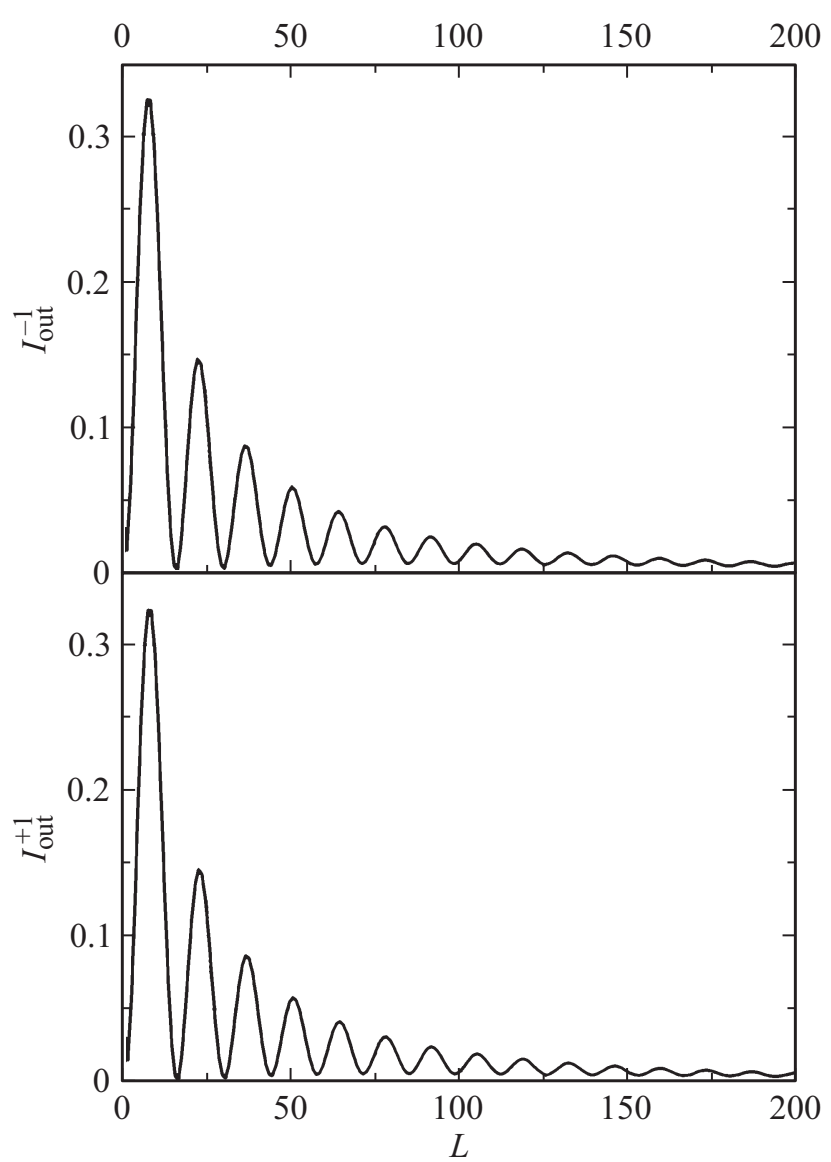

Рис. 5. Зависимость максимальной интенсивности $I_{\text {out }}$ в первых порядках дифракции от длины ячейки со средой $L$. Остальные параметры системы $\gamma_{1}=\gamma_{2}=\gamma_{3}=\gamma, \quad \Omega_{1}=\Omega_{3}=\gamma$, $\Delta_{1}=0.05 \gamma, \Delta_{2}=0, \Delta_{3}=0.15 \gamma, \varphi=0, \Lambda_{x} / \lambda_{2}=0.25, N=5$.

рядков (рис. 4,a). Более того, такое изменение приводит к увеличению интенсивности в центральном максимуме, полностью нивелируя собой фазовые эффекты решетки.

Увеличение отстроек $\Delta_{1}$ и $\Delta_{3}$ модулирующих полей также не приводит, согласно условию (14), к эффективной перекачке интенсивности пробного поля $\Omega_{2}$ в максимумы высших порядков (рис. $4, b$ ). Ситуация, когда наблюдаются условия $\frac{\Delta_{1}}{\Delta_{2}} \neq \frac{\Delta_{3}}{\Delta_{1}} ; \Delta_{1}, \Delta_{3} \ll \Omega_{1}, \Omega_{3}$, соответствует критическому падению интенсивности прошедшего поля, возникающего из-за ослабления когерентностей системы, создаваемых модулирующими полями $\Omega_{1}$ и $\Omega_{3}$ (рис. $4, b$ - штриховая, пунктирная, штрих-пунктирная и светло-серая линии). При выполнении условий $\frac{\Delta_{1}}{\Delta_{3}}=\frac{\Delta_{3}}{\Delta_{1}}$; $\Delta_{1}, \Delta_{3} \gg \Omega_{1}, \Omega_{3}$ картина перераспределения интенсивности пробного поля вновь меняется кардинально: вся интенсивность сосредоточена в центральном максимуме (рис. 4, $b$, темно-серый график).

Одним из ключевых параметров системы, влияющих на модуляцию функции трансляции (9) среды, является отстройка $\Delta_{2}$ самого пробного поля $\Omega_{2}$ от резонанса. Однако, как показывает анализ выражений (9) и рис. 4, $c$, даже незначительное отклонение значения $\Delta_{2}$ от резонанса приводит к критическому падению суммарной интенсивности на выходе из среды (график из точек). Физически это обусловлено тем, что нерезонансное пробное поле не попадает в окно прозрачности ЭИП, настроенное на резонансное значение. Таким образом, существенно увеличивается поглощение нерезонансно настроенного пробного поля, проходящего через модулированную среду.

Отметим, что ввиду вышеизложенного, учитывая предложенный физический размер ячейки $1.5 \mathrm{~m}$ и нерезонансно отстроенное пробное поле, экспериментально реализованная установка не сможет демонстрировать описанную в работе [21] картину перераспределения интенсивности на выходе из среды, так как большая часть интенсивности будет рассеиваться или поглощаться средой.

Влияние модулированной функции трансляции $T(x)$ существенно зависит от времени взаимодействия пробного поля $\Omega_{2}$ со средой, т.е. от длины ячейки $L$ вдоль оси $O z$. Однако, как видно на рис. 5, максимальное значение эффективности перераспределения в максимумы первого порядка соответствует значению $L=7$. Обусловливается такая зависимость тем, что при увеличении времени взаимодействия пробного поля $\Omega_{2}$ со средой начинают превалировать эффекты поглощения поля и преломления в высшие порядки. Таким образом, оптимальным значение длины, при котором наблюдается максимально значение интенсивности, является $L=7$ для выбранных параметров системы. Отметим, что данная зависимость рис. 5 демонстрирует эффективность перекачки интенсивности пробного поля $\Omega_{2}$ именно в максимумы первого порядка, и не несет информации о том, куда перераспределяется полная интенсивность.

\section{Заключение}

В работе исследована возможность создания ЭМИР в среде с четырехуровневой трипод-конфигурацией атомных состояний. Получена высокая эффективность перераспределения интенсивности пробного поля в максимумы первого порядка при сохранении компактности схемы. Исследовано влияние параметров системы на изменения дифракционной картины.

В области практического применения результаты исследований по данной тематике могут применяться в сфере телекоммуникаций для создания разного рода оптических переключателей (оптических маршрутизаторов), устройств хранения памяти, кроме того, эффект может применяться для контроля химических реакций.

\section{Финансирование работы}

Работа поддержана Министерством образования и науки РФ (проект 3.821.2014/K), грантом 074-U01 для лидирующих университетов РФ и грантом РФФИ 17-0200598 A. 


\section{Конфликт интересов}

Авторы заявляют, что у них нет конфликта интересов.

\section{Список литературы}

[1] Ling H.Y., Li Y., Xiao M. // Phys. Rev. A. 1998. V. 57. N 2. P. 1338.

[2] Mitsunaga M., Imoto N. // Phys. Rev. A. 1999. V. 59. N 6. P. 4773.

[3] Brown A.W., Xiao M. // Opt. Lett. 2005. V. 30. N 7. P. 699.

[4] Zhao L., Duan W., Yelin S.F. // Phys. Rev. A. 2010. V. 82. N 1. P. 013809.

[5] Moretti D., Felinto D., Tabosa J.W.R., Lezama A. // J. Phys. B: At. Mol. Opt. Phys. 2010. V. 43. N 11. P. 115502.

[6] Bajcsy M., Zibrov A.S., Lukin M.D. // Nature. 2003. V. 426. P. 638.

[7] Kuang S.-q., Wan R.-g., Kou J., Jiang Y., Gao J.-y. // J. Opt. Soc. Am. B. 2010. V. 27. N 7. P. 1518.

[8] Kozlov D.N., Bombach R., Hemmerling B., Hubschmid W. // Opt. Lett. 1997. V. 22. N 1. P. 46.

[9] Kuang S.-q., Wan R.-g., Du P., Jiang Y., Gao J.-y. // Opt. Express. 2008. V. 16. P. 15455.

[10] Wen J., Zhai Y.-H., Du S., Xiao M. // Phys. Rev. A. 2010. V. 82. N 4. P. 043814.

[11] Sandor N., Bakos J.S., Sörlei Zs., Djotyan G.P. // J. Opt. Soc. Am. B. 2011. V. 28. N 11. P. 2785.

[12] Zhang Y., Wang Z., Nie Z., Li C., Chen H., Lu K., Xiao M. // Phys. Rev. Lett. 2011. V. 106. N 9. P. 093904.

[13] Kuang S.-qi, Jin C.-S., Li C. // Phys. Rev. A. 2011. V. 84. N 3. P. 033831 .

[14] Xiao Z.-H., Shin S.G., Kim K. // J. Phys. B: At. Mol. Opt. Phys. 2010. V. 43. N 16. P. 161004.

[15] de Araujo L.E.E. // Opt. Lett. 2010. V. 35. N 17. P. 977.

[16] Carvalho S.A., de Araujo L.E.E. // Opt. Express. 2011. V. 19. N 3. P. 1936.

[17] Carvalho S.A., de Araujo L.E.E. // Phys. Rev. A. 2011. V. 83. N 5. P. 053825 .

[18] Wan R.-G., Kou J., Jiang L., Jiang Y., Gao J.-Y. // Phys. Rev. A. 2011. V. 83. N 3. P. 033824.

[19] Carvalho S.A., de Araujo L.E.E. // Phys. Rev. A. 2011. V. 84. N 4. P. 043850.

[20] Xie B., Cai X., Xiao Z.-H. // Opt. Com. 2012. V. 285. N 2. P. 133.

[21] Ya-Bin D., Yao-Hua G. // Chin. Phys. B. 2014. V. 23. N 7. P. 074204. 